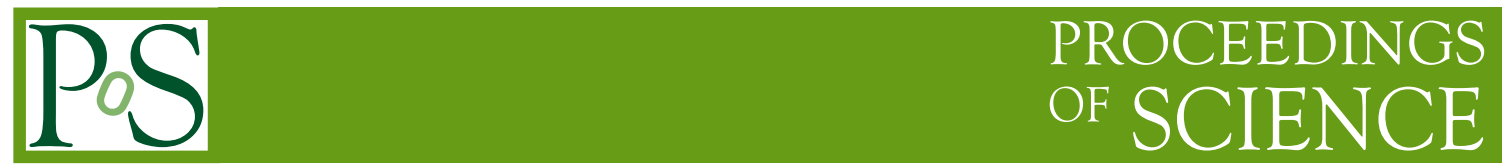

\title{
The ATLAS Muon Trigger Design and Performance
}

\author{
Alec Drobac*, on behalf of the ATLAS Collaboration \\ Physics and Astronomy Department, Tufts University \\ Medford, MA, United States of America \\ E-mail: alec.drobac@tufts.edu
}

\begin{abstract}
Muon triggers are essential for studying a variety of physics processes in the ATLAS experiment at the LHC, including Standard Model measurements and searches for new physics. The ATLAS muon trigger system consists of a hardware-based Level-1 trigger and a software-based HighLevel Trigger for reconstruction purposes. The muon triggers have been optimized during Run 2 to provide high efficiencies while keeping the trigger rate low. These proceedings give an overview of how ATLAS triggers on muons, recent improvements to the trigger system, the performance of the muon trigger based on Run 2 data, and the upgrades underway for Run 3.
\end{abstract}

The Eighth Annual Conference on Large Hadron Collider Physics-LHCP2020

25-30 May, 2020

online

\footnotetext{
${ }^{*}$ Speaker
} 
Alec Drobac

\section{Introduction}

The ATLAS experiment [1] uses a trigger system to select potentially interesting events out of the billions of proton-proton collisions which occur every second at the Large Hadron Collider (LHC). Many processes of interest to both Standard Model measurements and new physics searches involve muons in the final state, so it is critical to analyze events containing prompt muons (i.e. originating from the primary vertex). Therefore, a menu of muon triggers is rigorously maintained to maximize phase-space coverage within the allowed bandwidth. Presented here are a description of the trigger system performance in the high-luminosity environment of Run 2 and a summary of improvements in store for Run 3.

\section{Trigger System}

The ATLAS trigger system consists of two primary components: the hardware-based Level-1 (L1) trigger and the software-based High-Level Trigger (HLT). The L1 trigger receives events at the LHC bunch-crossing rate of around $40 \mathrm{MHz}$ and, using coarse-grained calorimeter and muon spectrometer information, passes Regions of Interest to the HLT at a maximum rate of $100 \mathrm{kHz}$. The HLT then uses full-granularity detector information to select events to be stored at an average rate of $1 \mathrm{kHz}$. The L1 topological processor (L1Topo), which can perform topological selections by combining kinematic information from multiple calorimeter and muon trigger objects, was commissioned during Run 2 in 2016 [2].

\section{Muon Spectrometer}

Muons are measured independently in the Inner Detector (ID) and Muon Spectrometer (MS) of the ATLAS detector; the latter is shown in Figure 1. In the barrel region $(|\eta|<1.05)$ of the MS, Resistive Plate Chambers (RPCs) provide the triggering at L1 while Monitored Drift Tubes (MDTs) provide precision tracking. Similarly, in the endcap region $(1.05<|\eta|<2.4)$, Thin Gap Chambers (TGCs) provide the

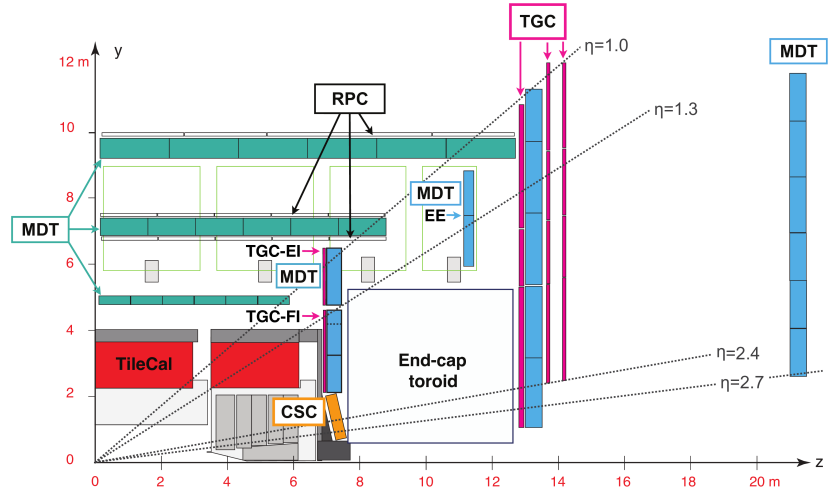

Figure 1: A quarter-section view of the Muon Spectrometer in a plane containing the beam axis [3]. L1 triggering while MDTs and Cathode Strip Chambers (CSCs) provide the tracking.

\section{Trigger Efficiency Studies}

Trigger efficiency measurements are performed using a tag-and-probe method on pairs of muons reconstructed offline from $Z$ or $J / \psi$ decays. By stringent selection of tag muons, an unbiased sample of probe muons on which to conduct trigger performance studies is produced. Tag muon candidates must pass quality requirements and have a transverse momentum $\left(p_{\mathrm{T}}\right)$ greater than the tag trigger threshold ( $28 \mathrm{GeV}$ in 2018). A tag candidate is also required to geometrically match the object which fired the tag trigger. The other muon in the pair is then a probe candidate; to test the trigger efficiency, a check is made to see if it matches the object which fired the probe trigger. 

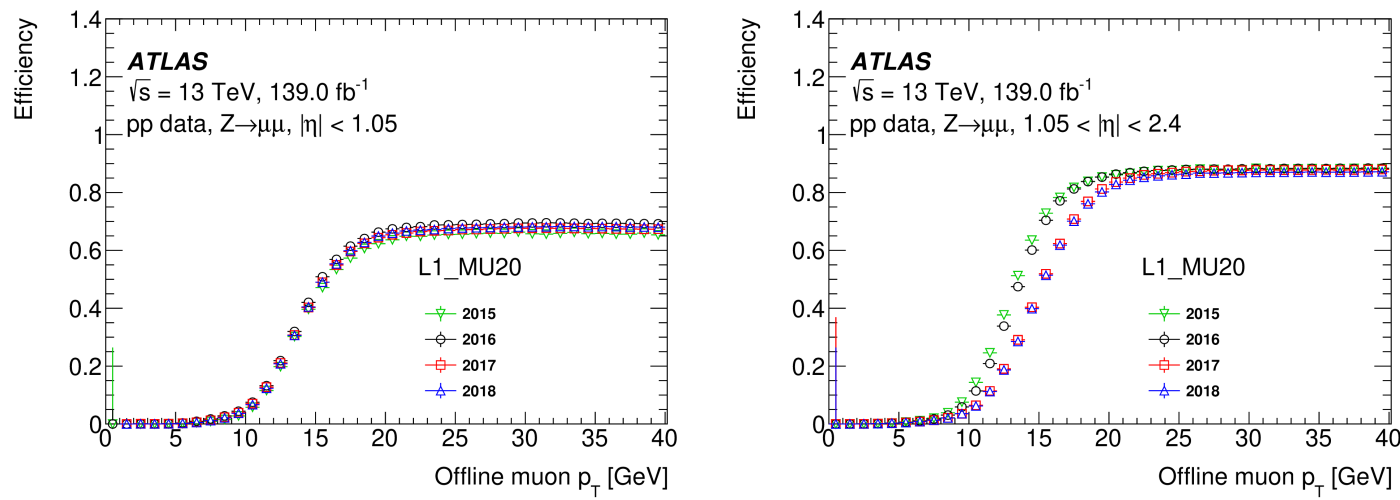

Figure 3: Efficiency of the L1_MU20 trigger as a function of the offline-reconstructed muon $p_{\mathrm{T}}$ in the barrel (left) and endcap (right) regions [3].

\section{Run 2 Performance}

The muon trigger system performance was consistent throughout Run 2 despite increasing event rates. Additional detector coincidence requirements ${ }^{1}$ helped to gradually reduce the L1 trigger rate, as shown in Figure 2. The HLT trigger rate was roughly constant during the entire run.

Figure 3 shows the trigger efficiency of the lowest unprescaled L1 trigger as a function of the probe muon $p_{\mathrm{T}}$ in the barrel (left) and endcap (right) regions. The trigger efficiency in the barrel plateaus at $\sim 70 \%$, significantly lower than the $\sim 90 \%$ plateau of the endcap efficiency, due to reduced geometrical coverage in the barrel region. In the endcap region, the shift in the turn-on curve from 2016 to 2017 is a result of the coincidence windows for low- $p_{\mathrm{T}}$ triggers being tightened.

Figure 4 shows an endcap trigger efficiency plot for the lowest unprescaled HLT trigger. The scale factor (SF), the ratio of measured to predicted efficiencies, is included in the lower panel; this is used to correct the muon trigger efficiency in simulations. The HLT trigger efficiencies were

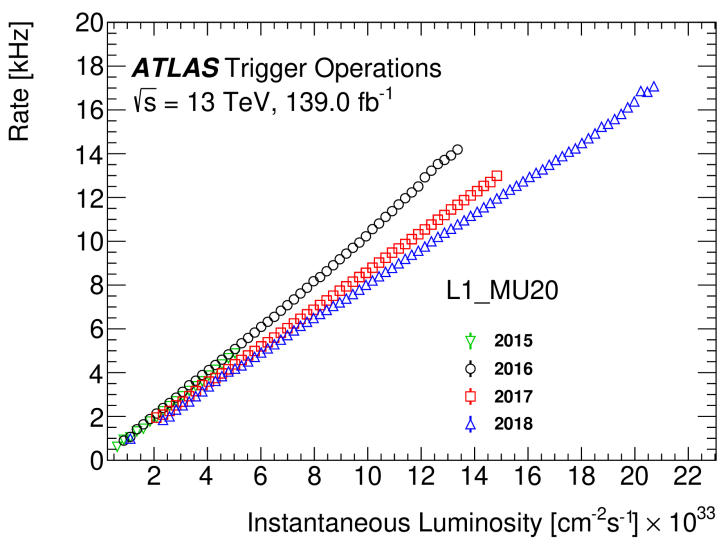

Figure 2: Event rate as a function of the instantaneous luminosity for the lowest unprescaled L1 trigger, L1_MU20 $\left(p_{\mathrm{T}}\right.$ threshold of $20 \mathrm{GeV}$ ), for each year of data taking during Run 2 [3].

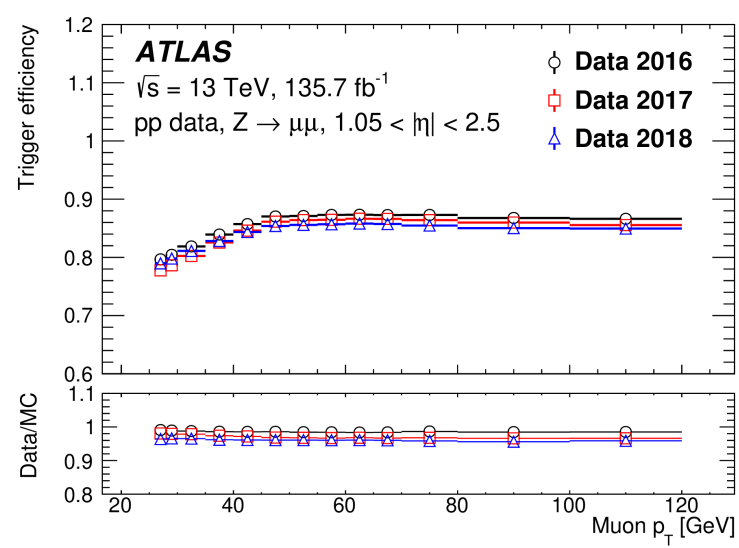

Figure 4: Efficiency as a function of the offlinereconstructed muon $p_{\mathrm{T}}$ for the lowest unprescaled HLT trigger, with a $p_{\mathrm{T}}$ threshold of $26 \mathrm{GeV}$, in the endcap region [3].

${ }^{1}$ These include a coincidence between the TGCs on the Big Wheel and those on the Small Wheel, and another between the Big Wheel TGCs and the tile hadronic calorimeter. This additional coincidence reduces contamination by charged particles originating from interactions between protons scattered at the beam pipe and the ATLAS toroidal magnets. 
kept stable throughout Run 2.

Overall, the efficiency of single-muon triggers for Run 2 was $\sim 68 \%(85 \%)$ in the barrel (endcap) regions; multi-muon trigger efficiencies (the products of the single-leg efficiencies but with looser L1 requirements) were even higher. In addition, the new L1Topo requirement significantly reduced the L1 trigger rate for the low- $p_{\mathrm{T}}$ triggers used extensively by the $B$-physics and Light States program, as shown in Figure 5a.

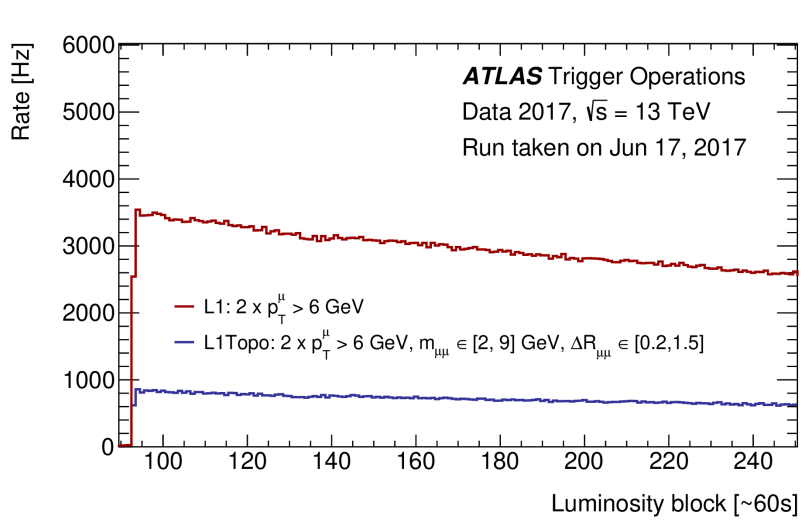

Figure 5a: L1 trigger rate as a function of the luminosity without (red) and with (blue) the L1Topo requirement. The rate is reduced by approximately a factor of 4 [3].

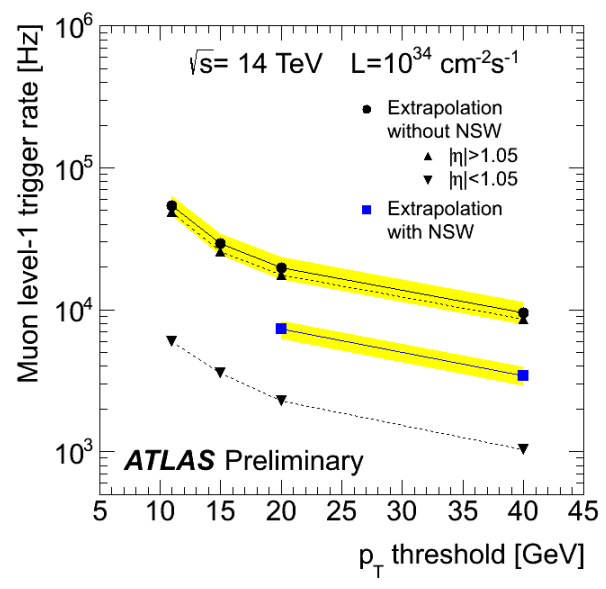

Figure 5b: Estimation of the Run 3 L1 trigger rates, extrapolated for $p p$ collisions at $\sqrt{s}=14 \mathrm{TeV}$, as a function of the $p_{\mathrm{T}}$ threshold [4].

\section{Upgrades for Run 3}

Several upgrades to the muon trigger system will be made to handle the even higher event rates of Run 3. The most significant change is the installation of the New Small Wheel (NSW) at $1.3<|\eta|<2.7$. The NSW's increased granularity should significantly decrease the L1 trigger rate, as the extrapolation in Figure 5b shows. Other upgrades include new RPC chambers at $1.0<|\eta|<1.3$ to further assist in rejecting fake muons and the migration of the HLT system to a multi-threaded CPU platform (which has been completed and is now undergoing validation) to minimize CPU and memory usage. These and other improvements to the muon trigger system will help ATLAS complete its upcoming physics program. 


\section{References}

[1] ATLAS Collaboration, The ATLAS Experiment at the CERN Large Hadron Collider, JINST 3 S08003 (2008).

[2] Arantxa Ruiz Martínez, on behalf of the ATLAS Collaboration, The Run-2 ATLAS Trigger System, ATL-DAQ-PROC-2016-003, https://cds.cern.ch/record/2133909.

[3] ATLAS Collaboration, Performance of the ATLAS muon triggers in Run 2, arXiv:2004.13447.

[4] ATLAS Collaboration, Estimation of muon level-1 trigger rate extrapolated for $\sqrt{s}=14 \mathrm{TeV}$ by emulating impact of new small wheel detector, https://twiki.cern.ch/twiki/bin/view/AtlasPublic/MuonTriggerPublicResults. 